\title{
Neurinomas intramedulares. Presentación de dos casos de un tumor infrecuente
}

L.M. Bernal-García; J.M. Cabezudo-Artero; M. Ortega-Martínez; L.F. Porras-Estrada; I. Fernández-Portales; L.F. Ugarriza-Echebarrieta; M. Molina-Orozco y *J.J. Pimentel-Leo

Servicios de Neurocirugía y de *Anatomía Patológica. Hospital Universitario Infanta Cristina. Badajoz.

\section{Resumen}

Los schwannomas intramedulares son tumores benignos derivados de células de Schwann localizadas en el parénquima medular. Son muy infrecuentes, constituyendo entre el 0,3 y el $1,5 \%$ de los schwannomas espinales. Existen varias teorías que intentan explicar la presencia de células de Schwann intramedulares a partir de las que derivarían estas neoplasias. La sintomatología no es específica, siendo el dolor el síntoma más frecuente, pudiéndose añadir, además, cualquier signo de afectación medular según el nivel de localización. En las pruebas de neuroimagen, la sospecha de otras lesiones intramedulares de origen glial más frecuentes hace que el diagnóstico de schwannoma intramedular sea postquirúrgico la mayoría de las veces. El tratamiento es quirúrgico, pudiendo llevarse a cabo, habitualmente, una resección completa.

En este artículo presentamos dos casos de pacientes intervenidas quirúrgicamente de sendas lesiones intramedulares, que resultaron ser neurinomas. Hacemos una revisión de otros casos publicados en la literatura, exponiendo las teorías existentes acerca del origen de estos tumores poco frecuentes.

PALABRAS CLAVE: Célula de Schwann. Neurinoma intramedular. Schwannoma. Tumor espinal. Tumor intramedular.

Intramedullary schwannomas. Report of two cases

\section{Summary}

Intramedullary schwannomas are benign tumors that arise from clusters of Schwann cells embedded in the medullary parenchyma. They are very rare and account for 0.3 to $1.5 \%$ of all spinal schwannomas. It is not known for certain why this happens, and several theories have been put forward. They do not exhibit

Recibido: 8-07-098. Aceptado: 7-10-09 any characteristic clinical picture, being pain the most frequent symptom. Imaging is also not specific and the correct diagnosis is attained most of the times after pathological examination. The goal of treatment must be complete removal, which is curative.

We are presenting two patients in whom an intramedullary tumor was removed. The true nature of the lesions was suspected during the operation, but only after pathological examination was confirmed. A review of the literature has been undertaken, with special attention to the theories about the origin of these uncommon tumors.

KEY WORDS: Schwann cell. Intramedullary neurinoma. Schwannoma. Spinal tumor. Intramedullary tumor.

\section{Introducción}

Los schwannomas (también llamados neurinomas o neurilemomas) $)^{1,9}$ son tumores benignos, de lento crecimiento, que derivan de las células de Schwann ${ }^{1,6,21}$. Pueden ocurrir en cualquier lugar del sistema nervioso, central o periférico, donde se encuentren, de forma normal o aberrante, dichas células ${ }^{21}$.

Estos tumores constituyen el $30 \% 0^{1,3,4,6,7,9,22}$ de las neoplasias espinales primarias. Suelen ser lesiones de localización intradural extramedular ${ }^{1-3,15,21}$, en relación con las raíces nerviosas ventrales 0 , más frecuentemente, dorsales ${ }^{21}$ de la médula. Su presentación como tumores intramedulares es muy infrecuente, ya que sus células de origen no se encuentran, normalmente, en el parénquima medular ${ }^{1,2,9,4-}$ 7,11,22. Los schwannomas intramedulares suponen tan sólo el $0,3-1,5 \% \%^{1,4,6,7,15,22}$ de los schwannomas espinales y el $0,3 \%$ de todas las neoplasias intraespinales ${ }^{9,4,16}$. Se han descrito varias teorías referentes al origen intramedular de los mismos, sin que ninguna tenga la aceptación universal.

El conocimiento del neurinoma intramedular es de gran

Abreviaturas. EEII: extremidades inferiores. GFAP: proteina glial fibrilar ácida. NF: neurofibromatosis. RM: resonancia magnética. TC: tomografía computerizada. 

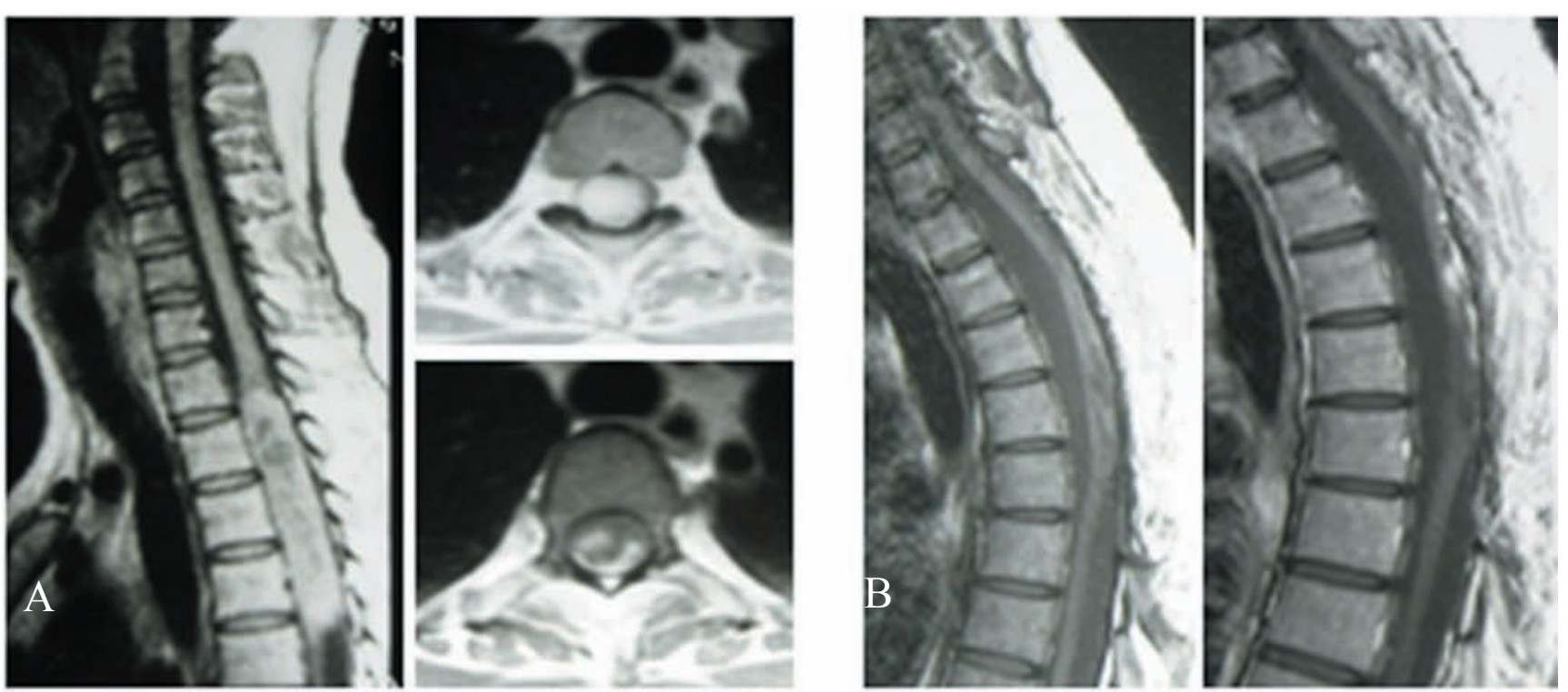

Figura 1. A: RM en secuencia T1 con contraste del caso 1, en la que se aprecia una tumoración intramedular a nivel dorsal, con importante realce con el contraste paramagnético. B: RM de control a los 8 años de la intervención. Se aprecia una atrofia de la médula a ese nivel con una captación de contraste que ha permanecido estable en todos estos años.

interés, ya que se plantea el diagnóstico diferencial con otras neoplasias intramedulares como el ependimoma o el astrocitoma ${ }^{2,22}$, con las que existen diferencias en cuanto al tratamiento y el pronóstico.

En este artículo presentamos dos casos de pacientes tratadas en nuestro servicio de lesiones intramedulares que resultaron ser schwannomas. Hacemos una revisión de otros casos publicados, exponiendo las diferentes teorías del origen y otros aspectos del diagnóstico y tratamiento de estas lesiones.

\section{Casos clínicos}

\section{Caso clínico 1}

Mujer de 35 años de edad, sin antecedentes personales de interés, con clínica de tres años de evolución de dolor en miembro inferior derecho, acompañado de parestesias y torpeza progresivas, junto a alteraciones esfinterianas en forma de urgencia miccional. A su ingreso presentaba una paraparesia de predominio derecho, con hiperreflexia osteotendinosa de extremidades inferiores (EEII), clonus aquíleo y signo de Babinski bilateral; la exploración sensitiva mostró afectación de la sensibilidad posicional y termoalgésica con nivel D3 derecho y D5 izquierdo.

La resonancia magnética (RM) de columna dorsal realizada (Figura 1) mostró un engrosamiento de la médula dorsal a nivel D1-D5 por la presencia de una lesión que era isointensa en las imágenes potenciadas en T1, hiperintensa en las imágenes potenciadas en T2, y con captación bastante homogénea de contraste paramagnético, compatible todo ello con la presencia de un tumor intramedular.
La paciente fue intervenida quirúrgicamente, realizándose una laminectomía desde D1 hasta D5, apertura dural y mielotomía por surco medio posterior y resección macroscópicamente completa de la lesión, que presentaba buena delimitación con respecto al tejido sano, quedando una médula muy adelgazada.

La lesión fue informada como un schwannoma, con la presencia de células alargadas con núcleos fusiformes con tendencia a disponerse en empalizada en algunas zonas (Figura 3). En el estudio con técnicas inmunohistoquímicas se constató positividad para la proteína $\mathrm{S} 100$ y negatividad para la proteína glial fibrilar ácida (GFAP).

El postoperatorio fue satisfactorio, sin presentar complicaciones añadidas.

Tras 17 años de seguimiento, presenta un piramidalismo de EEII y una afectación de la sensibilidad posicional que le provoca una leve ataxia de la marcha; a pesar de lo cual realiza una vida funcionalmente activa y completamente independiente. Recuperó la alteración de la sensibilidad termoalgésica y el control esfinteriano.

En las sucesivas RM de control (Figura 1) se comprobó la resección completa de la lesión. Se apreció una captación de contraste en la parte posterior de la médula en el nivel intervenido que se ha mantenido estable durante estos años, achacándose a la presencia de fibrosis y cambios postquirúrgicos.

\section{Caso clínico 2}

Mujer de 18 años de edad en seguimiento por Neurofibromatosis (NF) tipo II. Se diagnosticaron mediante RM múltiples lesiones a lo largo del canal raquídeo y lesiones 

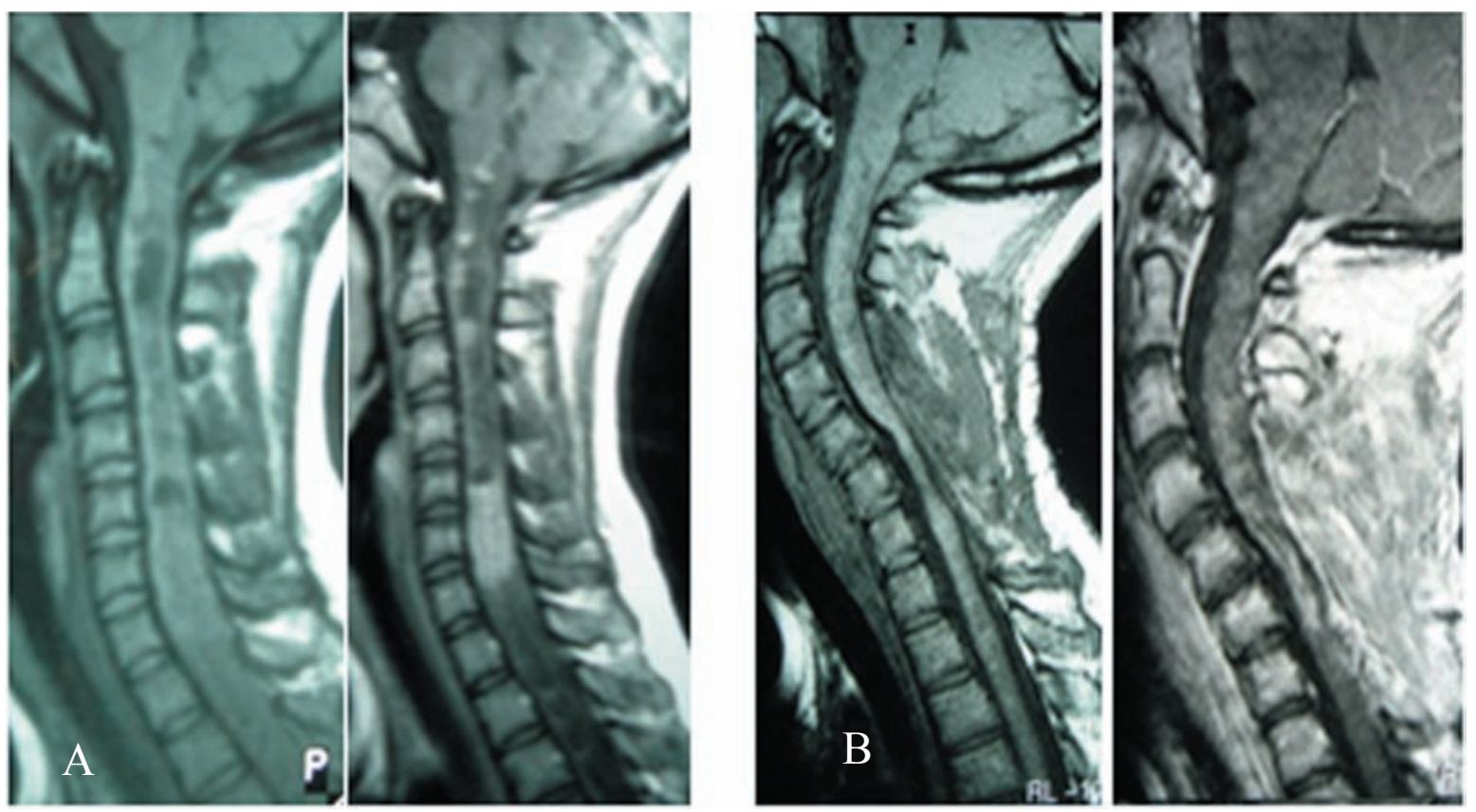

Figura 2. A: RM sin y con contraste del caso 2, que muestra un engrosamiento bulbomedular hasta C7, con áreas hipointensas en T1. Tras la administración de contraste se aprecian varias lesiones nodulares, la mayor de ellas localizada a nivel C5-C7, con captación intensa y homogénea de contraste y quiste en región polar superior, bastante bien delimitada del parénquima medular adyacente. B: RM sin y con contraste de control actual, que muestra la presencia de captaciones nodulares a lo largo de la médula cervical similares a estudios previos, con atrofia focal de la médula a nivel de la localización donde se encontraba el neurinoma intervenido.

$\mathrm{H} / \mathrm{E}$

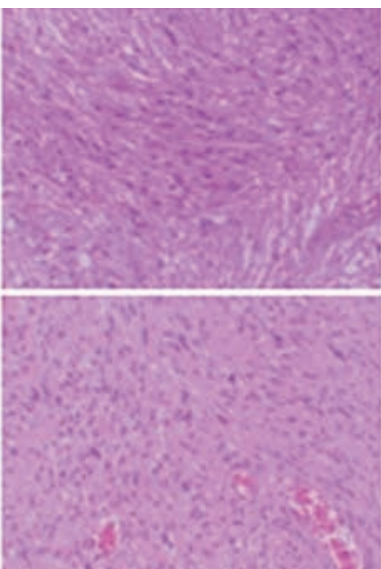

GFAP

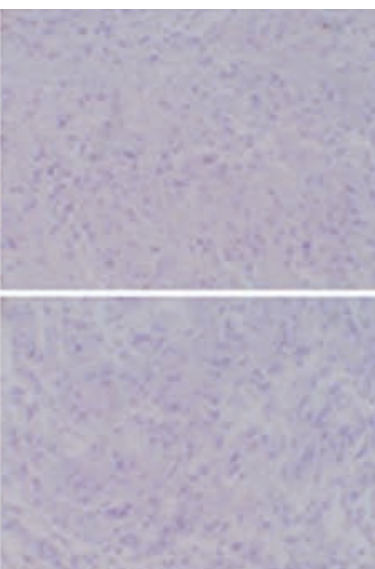

Proteína S-100

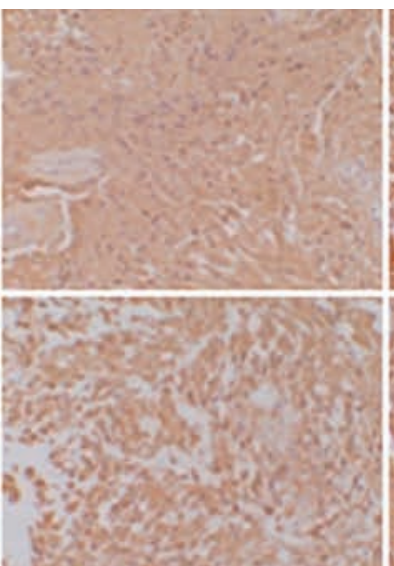

Vimentina

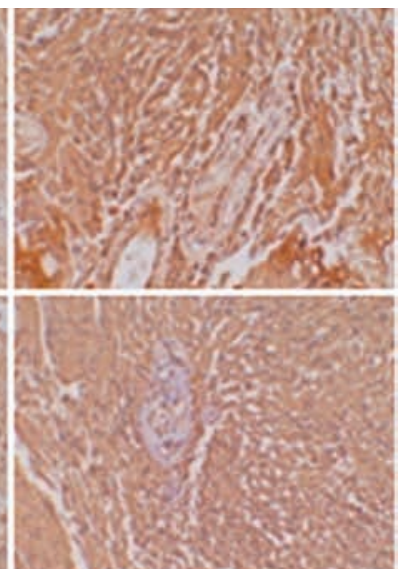

Figura 3. Preparaciones histológicas (x20) de ambos casos. Con Hematoxilina/Eosina, se aprecian células alargadas, con núcleos fusiformes que tienden a disponerse en empalizada. En las demás preparaciones se aprecia negatividad para GFAP y positividad para Proteina S-100 y vimentina.

intracraneales de ambos ángulos pontocerebelosos compatibles con neurinomas del acústico.

La paciente presentaba clínica de dos años de evolución de inestabilidad en la marcha y torpeza en EEII que fue progresando hasta una paraparesia de predominio derecho. Posteriormente se vio afectado el miembro superior dere- cho, presentando una deformidad de la mano con muñeca en extensión y dedos en flexión. Los reflejos osteotendinosos estaban exaltados en las extremidades inferiores y había clonus aquíleo y signo de Babinski bilateral. En la exploración de la sensibilidad se objetivó afectación de la sensibilidad posicional y termoalgésica con nivel sensitivo C6 
Tabla I

Casos de neurinomas intramedulares descritos en la literatura

\begin{tabular}{lcclclc}
\hline Autor & $N^{\text {o de casos }}$ & Edad (años) & Sexo & Con NF & Localización & Duración de los síntomas \\
\hline Siddiqui y cols. & 66 & $\begin{array}{l}\text { Rango: } 9-75 \\
\text { Media: } 41,5\end{array}$ & $\begin{array}{c}\text { Varón/Mujer } \\
1,5 / 1\end{array}$ & 11 & $\begin{array}{l}\text { Cervical: } 65 \% \\
\text { Dorsal: } 22 \% \\
\text { Lumbar: } 10 \%\end{array}$ & 3 meses-20 años \\
\hline Santaguida y cols. & 1 & 35 & Varón & No & Cervical & 10 meses \\
\hline El Malki y cols. & 1 & 40 & Mujer & No & Cervical & 7 años \\
\hline Mukerji y cols. & 1 & 8 & Varón & No & Cervical & 6 meses \\
\hline Bernal y cols. & 2 & 35 & $\begin{array}{l}\text { Mujer } \\
\text { Mujer }\end{array}$ & $\begin{array}{c}\text { No } \\
1\end{array}$ & $\begin{array}{l}\text { Dorsal } \\
\text { Cervical }\end{array}$ & $\begin{array}{l}3 \text { años } \\
2 \text { años }\end{array}$ \\
\hline
\end{tabular}

NF: neurofibromatosis

derecho y C5 izquierdo.

La RM de columna (Figura 2) presentaba un engrosamiento bulbomedular hasta $\mathrm{C} 7$, con áreas hipointensas en $\mathrm{T} 1$ e hiperintensas en T2 en su interior. Tras la administración de contraste paramagnético se apreciaron varias lesiones nodulares, la mayor de ellas localizada a nivel C5-C7, con captación intensa y homogénea de contraste y quiste en región polar superior, bastante bien delimitada del parénquima medular adyacente. Además, se apreciaron varias lesiones intramedulares con componentes quísticos confluentes en regiones cervical alta y bulbar.

La paciente fue intervenida, realizándose una laminectomía desde $\mathrm{C} 4$ a $\mathrm{C} 7$, apertura dural y mielotomía por surco medio posterior, con extirpación macroscópicamente completa de la lesión. Se apreció buen plano de disección con el tejido medular circundante, quedando una médula tremendamente adelgazada.

El estudio histopatológico mostró una lesión con células alargadas y núcleos fusiformes. Las tinciones inmunohistoquímicas mostraron positividad para la proteína S100 y negatividad para la GFAP, siendo informada como schwannoma. (Figura 3).

En el postoperatorio inmediato presentó una fístula de líquido cefalorraquídeo que se resolvió con tratamiento conservador mediante la colocación de un drenaje lumbar, y una infección superficial de la herida quirúrgica que fue tratada con antibióticos. Tras tratamiento rehabilitador intenso, obtuvo mejoría de sus déficits, quedando con una marcha pareto-espástica, con piramidalismo y una afectación de la sensibilidad propioceptiva. En las RM de control de columna cervical (Figura 2) se apreciaron los cambios postquirúrgicos, con una marcada atrofia medular a nivel cervical bajo y un engrosamiento bulbar y de la médula cervical alta secundaria a la presencia de las lesiones intramedulares ya conocidas previamente y que se han mantenido estables hasta el momento actual.

\section{Discusión}

\section{Aspectos clínicos}

En 1931, Kernohan et al. ${ }^{12}$ publicaron el primer caso de neurinoma intramedular. Desde entonces hasta ahora sólo se han publicado 69 casos de tumores de este tipo, aparte de los dos nuestros. Efectivamente, los neurinomas intramedulares son tumores muy infrecuentes (entre 0.3$1.5 \%$ de los schwannomas espinales ${ }^{1,4,6,7,15,22}$ ), debido a la ausencia de células de Schwann en la médula.

Dada la naturaleza benigna de los neurinomas y su lento crecimiento, los síntomas suelen ser de larga evolución ${ }^{1,2}$. La clínica depende del nivel de la lesión. El dolor es el síntoma más frecuente ${ }^{11,15}$, pero además puede haber síntomas y signos de afectación motora, sensitiva y esfinteriana.

En los 71 casos revisados (Tabla I), se observa una relación varón/mujer de aproximadamente 1,5/1, con edades comprendidas entre los 8 y los 75 años. La duración de la sintomatología hasta el diagnóstico va desde los 3 meses a los 20 años, y fue diferente según la localización, siendo el dolor lo más frecuente. En cuanto al nivel medular, la localización cervical fue la más frecuente, seguida de la dorsal.

\section{Asociación con NF}

Las neoplasias intramedulares no han sido asociadas explícitamente con la NF. Sin embargo, Lee et al. ${ }^{13}$ consi- 
deran improbable que esta asociación no exista y señalan que esa aparente falta de asociación se deba a una aberración estadística, debido a la dificultad de relacionar un tumor raro, como son los tumores intramedulares, con un síndrome raro, como es la NF. Ellos publican una serie de 9 pacientes con NF y tumores intramedulares, uno de ellos con un neurinoma intramedular. Conti et al. ${ }^{5}$ refieren que los neurinomas intramedulares no deben ser considerados una rareza en la enfermedad de Von Recklinghausen. Publican una serie de 179 neurinomas espinales en 152 pacientes. De éstos había 11 pacientes afectados de NF. Hubo 3 neurinomas intramedulares en toda la serie y uno de ellos en una paciente con NF tipo 2. Entre los 71 casos de neurinomas intramedulares aquí revisados hay 12 pacientes afectos de neurofibromatosis, 5 de ellos con NF tipo 1 y 7 con NF tipo 2.

\section{Patogénesis}

Son varias las teorías que intentan explicar la presencia de las células de Schwann en el parénquima medular para postular el origen de estos tumores:

- Rammamurthi et al. ${ }^{18}$ proponen como causa una alteración en la embriogénesis, con invaginación hacia el interior del tubo neural de células de la cresta neural, que más tarde podrían diferenciarse en células de Schwann, que podrían sufrir posteriormente, y en cualquier momento, una transformación neoplásica.

- Hughes y Brownell ${ }^{10}$ se refieren a la presencia de células de Schwann intramedulares aberrantes, a menudo asociadas a cambios inflamatorios crónicos de la médula espinal.

- Riggs y Clary ${ }^{19}$ apuntan como origen de estos tumores las células de Schwann del plexo nervioso perivascular intramedular.

- Waggener ${ }^{24}$ propone la transformación de elementos ectodérmicos piales, en célula de Schwann.

- Mason y Keigher ${ }^{14}$ postulan el crecimiento neoplásico de la célula de Schwann de la raíz dorsal localizada en el "área crítica", en el punto donde la raíz posterior pierde su vaina de mielina para atravesar la piamadre, creciendo la tumoración de forma centrípeta.

Esta última teoría parece ser la que mejor explica la presencia de neurinomas intramedulares con componente extramedular, como es el caso publicado por Innocenzi et $\mathrm{al}^{11}$.

\section{Estudios diagnósticos}

Como apuntan Herregodts et al. ${ }^{9}$, los estudios de radiología simple pueden contribuir en el estudio de lesiones intrarraquídeas, entre ellas los tumores intramedulares, dando, en ocasiones, signos indirectos de la presencia de un tumor espinal como el aumento del diámetro ánteroposterior del canal espinal o el ensanchamiento del agujero de conjunción por horadamiento de los pedículos. Aunque en los tumores intramedulares la radiología simple no suele mostrar alteraciones.

La TC puede ser útil en el diagnóstico si no se cuenta con $\mathrm{RM}^{3,8}$.

Si bien la mielografía fue la técnica más utilizada en el diagnóstico de estos tumores ${ }^{2}$, actualmente es la RM la prueba de elección ${ }^{2-4,7}$. La administración de contraste paramagnético permite delimitar la tumoración, diferenciar el edema medular, descartar afectación ósea, y localizar el nivel espinal donde asienta la lesión ${ }^{9,11}$. Los schwannomas intramedulares suelen ser iso o hipointensos en $\mathrm{T} 1 \mathrm{y}$ moderadamente hiperintensos en $\mathrm{T} 2$, aunque la intensidad de la señal en la RM depende de las características macroscópicas (componentes sólidos o quísticos) y de la composición histológica (prevalencia de tejido Antoni A o Antoni B, presencia de melanina en las variantes melanóticas). Generalmente los tumores están bien delimitados y el edema perilesional es moderado. La captación de contraste es bastante homogénea e intensa, aunque puede ser parcheada en los tumores con componente quístico y/o necrótico. La siringomielia asociada es infrecuente ${ }^{4}$. Estas características descritas no permiten la distinción de estos tumores de otras neoplasias intramedulares más frecuentes, como son los astrocitomas o los ependimomas ${ }^{2,4,15}$. Se puede sospechar su diagnóstico preoperatorio en los casos en los que la RM muestre un componente extramedular predominante o cuando el tumor intramedular se continúe con una raíz nerviosa engrosada ${ }^{4}$.

\section{Estudio histopatológico}

El análisis histológico es el que nos dará el diagnóstico definitivo, siendo necesarias pruebas de inmunohistoquímica para la confirmación y permitir su diferenciación con otros tumores intramedulares. El schwannoma típico está compuesto por una proliferación de células de Schwann que muestran dos patrones: uno celular denso, llamado tejido tipo A de Antoni, y otro donde las células están separadas por una matriz laxa, llamado tejido tipo $\mathrm{B}$ de Antoni. El tejido tipo A de Antoni está formado por fascículos compactos de células alargadas bipolares, con núcleos fusiformes, que pueden formar agrupaciones en empalizada conocidas con el nombre de cuerpos de Verocay. Estas estructuras se alternan con regiones fibrilares anucleadas. Los cuerpo de Verocay, aunque son muy característicos de esto tumores, no son necesarios para hacer un diagnóstico de schwannoma. El tejido tipo B de Antoni es un tejido más laxo, con tendencia a la degeneración mucinosa, presencia de núcleos celulares redondeados y presencia de células xantomatosas que son responsables del color amarillento de estos tumores ${ }^{17,20}$.

Las tinciones inmunohistoquímicas de los neurinomas muestran positividad a la proteína S-100 y también a la 
Leu-7 y la vimentina. La tinción para proteína S-100 y la ausencia de tinción para la GFAP permiten el diagnóstico diferencial con los gliomas intramedulares. Los ependimomas muestran positividad a la GFAP, que suele ser muy marcada a nivel de las pseudorrosetas perivasculares, y pueden mostrar positividad focal a la vimentina y la proteína $\mathrm{S}-100^{20,23}$.

\section{Tratamiento}

El tratamiento de elección debe ser la exéresis quirúrgica. Puesto que la mayoría son tumores benignos, el objetivo primordial es la resección completa ${ }^{3,6,7,11,15,22}$. La exéresis subtotal provocaría su recidiva ${ }^{16}$. Como son tumores extremadamente raros, suele ser difícil distinguirlos preoperatoriamente de los gliomas ${ }^{9,15,5}$. Los gliomas son tumores infiltrantes y el objetivo de conseguir su resección completa puede condicionar una cirugía demasiado agresiva, que puede añadir déficits neurológicos sin obtener ningún beneficio ${ }^{5,15}$. Por ello es de suma importancia el análisis histológico intraoperatorio ${ }^{4,9,15,16}$.

La exéresis completa de un tumor intramedular (incluyendo el schwannoma) sin empeorar los déficits neurológicos previos, es un reto en la práctica neuroquirúrgica ${ }^{22}$. La técnica microquirúrgica y el uso juicioso del aspirador ultrasónico permiten obtener este objetivo con mínima retracción del tejido medular sano. Como apuntan Colosimo et al. ${ }^{4}$, el uso intraoperatorio de los ultrasonidos permite averiguar la localización exacta del tumor y guiar la extensión de la mielotomía, limitando la agresión quirúrgica de la médula a la mínima imprescindible para la exéresis tumoral. También el uso de potenciales evocados somatosensoriales y motores es una herramienta de gran ayuda. Aunque como refieren Siddiqui et al..$^{22}$ no está disponible en todos los centros. Tanto en su caso como en el nuestro, no existe la disponibilidad de esta técnica para la monitorización intraoperatoria. En cualquier caso, la consistencia de este tipo de tumores y la buena delimitación de su cápsula con respecto al parénquima medular, junto con una depurada técnica microquirúrgica, permiten obtener resultados óptimos en la exéresis completa de los neurinomas intramedulares.

\section{Conclusiones}

Los schwannomas intramedulares constituyen un grupo raro de tumoraciones espinales. Son benignos, de crecimiento lento, por lo que se puede retrasar su diagnóstico incluso hasta años desde el comienzo de los síntomas. La $\mathrm{RM}$ es fundamental para el diagnóstico, pero no permite distinguirlos de otros tumores intramedulares más frecuentes, como los astrocitomas o los ependimomas. Por eso es muy importante el diagnóstico intraoperatorio. El tratamiento es quirúrgico, y su exéresis completa debe cons- tituir el objetivo terapéutico de estos tumores infrecuentes pero potencialmente curables.

\section{Bibliografía}

1. Acciarri, N., Padovani, R. and Riccioni, L.: Intramedullary melanotic schwannoma. Report of a case and review of the literature. Br J Neurosurg 1999; 13: 322-325.

2. Binatli, O., Ersahin, Y., Korkmaz, O., Bayol, U.: Intramedullary schwannoma of the spinal cord: A case report and review of the literature. J Neurosurg Sci 1999; 43: 163-168.

3. Carrillo-Esper, R., Solís Maldonado, G., Trujillo, V., Téllez-Morales, MA., Salinas-Ruiz, S.: Schwannoma intramedular cervical. Cir Ciruj 2001; 69: 5-7.

4. Colosimo, C., Cerase, A., Denaro, L., Maira, G., Greco, R.: Magnetic resonance imaging of intramedullary spinal cord schwannomas. J Neurosurg (Spine) 2003; 99: 114-117.

5. Conti, P., Pansini, G., Mouchaty, H., Capuano, C., Conti, R.: Spinal neurinomas: retrospective analysis and long-term outcome of 179 consecutively operated cases and review of the literature. Surg Neurol 2004; 61: 35-44.

6. Darwish, B.S., Balakrishnan, V., Maitra, R.: Intramedullary ancient schwannoma of the cervical spinal cord: case report and review of literature. J Clin Neurosci 2002; 9: 321323.

7. El Malki, M., Bertal, A., Sami, A., Ibahoin, A., Lakhdar, A., Naja, A., et al.: Schwannome intramédullaire. À propos d'un case. Neurochirurgie 2005; 51: 19-22.

8. Gelabert, M., García, A., Conde, C., Bollar, A., Martínez, R., Reyes, F.: Intramedullary spinal neurofibroma diagnosed with computed tomography: report of one case. Neurosurgery $1985 ; 16$ : 543-545.

9. Herregodts, P., Vloeberghs, M., Schmedding, E., Goosens, A., Stadnik, T., D'Haens, J.: Solitary dorsal intramedullary schwannoma. Case report. J Neurosurg 1991; 74: 816-820.

10. Hughes, J.T., Brownell, B.: Aberrant nerve fibers within the spinal cord. J Neurol Neurosurg Psychiatry 1963; 26: $528-534$.

11. Innocenzi, G., Cervoni, I., Caruso, R.: Neurinoma cervical intramidolare. Descrizione di un caso e revisione della letteratura. Minerva Chir 1997; 52: 679-682.

12. Kernohan, J.W., Terae, S., Hida, K., Adson, A.W.: Intramedullary tumor of the spinal cord. Arch Neurol Psychiat 1931; 25: 679-699.

13. Lee, M., Rezai, A., Freed, D., Epstein, F.: Intramedullary spinal cord tumors in neurofibromatosis. Neurosurgery 1996 ; 38: 32-37.

14. Mason, T.H., Keigher, H.A.: Intramedullary spinal neurilemmoma. J Neurosurg 1968; 29: 414-416.

15. Mukerji, G., Sherekar, S., Yadav, Y.R., Chandrakar, S.K., Raina, V.K.: Pediatric intramedullary schwannoma without neurofibromatosis. Neurol India 2007; 55: 54-56. 
16. O'Brien, D.F., Farrell, M., Fraher, J.P., Bolger, C.: Schwann cell invasion of the conus medullaris: case report. Eur Spine J 2003; 12: 328-331.

17. Ortiz Hidalgo, C.: José Verocay. Neurinomas y cuerpos de Verocay y otras contribuciones a la medicina. Rev Neurol 2004; 39: 487-491.

18. Ramamurthi, B., Anguli, V.C., Iyer, C.G.S.: A case of intramedullary neurinoma. J Neurol Neurosurg Psychiatry 1958; 21: 92-94.

19. Riggs, H.E., Clary, W.U.: A case of intramedullary sheath cell tumor of the spinal cord: Consideration of vascular nerves as a source of origin. J Neuropathol Exp Neurol 1957; 16: 332-336.

20. Rodríguez de Lope, A., González Llanos, Fernández de Mesa, F.: Tumores schwannianos intrarraquídeos. En Tumores raquimedulares. Grupo de Neuro-oncología de la Sociedad Española de Neurocirugía. Ed. Centro de Estudios Ramón Areces. Madrid 2007. p. 191-201.

21. Santaguida, C., Sabbagh, A.J., Guiot, M.C., Del Maestro, R.F.: Aggressive intramedullary melanotic schwannoma: case report. Neurosurgery 2004; 55: E1430-E1434.

22. Siddiqui, A.A., Dr and Shah, A.A.: Complete surgical

\section{Comentario al trabajo Neurinomas intramedulares. Pre- sentación de dos casos de un tumor infrecuente de L.M. Bernal García y cols.}

Los autores describen dos nuevos casos en los que la histología de la neoplasia intramedular extirpada es la característica del schwannoma. Una de las pacientes reunía criterios explícitos coincidentes para el diagnóstico de neurofibromatosis tipo 2, mientras que en la descripción de la otra paciente no se hace referencia al resultado de la aplicación de técnicas de imagen para el rastreo de esta neurofibromatosis, por lo que no se aclara si se realizó este rastreo. De la revisión de las imágenes histológicas no caben dudas de que efectivamente se trata de schwannomas y el resultado terapéutico ha sido muy bueno, teniendo en cuenta el grado de afectación neurológica preoperatoria y la localización y extensión de los tumores. La localización intramedular de un schwannoma es un fenómeno muy infrecuente, que en la literatura se describe en una tasa aproximada del $1 \%$ de los schwannomas raquídeos y, por tanto, algo a considerar como evento excepcional en el diagnóstico de los tumores intramedulares. En el artículo revisado se mencionan 71 casos descritos en la literatura hasta la actualidad. Se revisan adecuadamente las hipótesis histogenéticas, el diagnóstico por imagen y las peculiaridades del tratamiento. Sin embargo, en la revisión bibliográ- excision of intramedullary schwannoma at the craniovertebral junction in neurofibromatosis type-2. Br J Neurosurg 2004; 18: 193-196.

23. Vaquero Crespo, J.: Ependimomas de la médula espinal. En Tumores raquimedulares. Grupo de Neurooncología de la Sociedad Española de Neurocirugía. Ed. Centro de Estudios Ramón Areces. Madrid 2007. p. 213230 .

24. Waggener, J.D.: Ultrastructure of benign peripheral nerve sheath tumours. Cancer 1966; 19: 699-709.

Bernal-García, L.M.; Cabezudo-Artero, J.M.; OrtegaMartínez, M.; Porras-Estrada, L.F.; Fernández-Portales, I.; Ugarriza-Echebarrieta, L.F.; Molina-Orozco, M.; Pimentel-Leo, J.J.: Neurinomas intramedulares. Presentación de dos casos de un tumor infrecuente. Neurocirugía 2010; 21: 232-239.

Correspondencia: Dr. Luis Miguel Bernal García. Servicio de Neurocirugía. Hospital Universitario Infanta Cristina. Avenida de Elvas s/n. 06006 Badajoz.

E-mail: lumi_bega@hotmail.com fica hay una definición incompleta de los casos referidos en cuanto a su asociación ó no con las neurofibromatosis: mientras en unos artículos esta asociación se considera una casualidad, en otros se hace expresión de su mayor frecuencia de la esperada en pacientes afectos de enfermedad de von Recklinghausen y de neurofibromatosis, tipos 1 ó 2. En la literatura médica además, hasta muy recientemente, se han utilizado indistintamente y con frecuencia erróneamente los términos referidos a estas dos enfermedades, e incluso los referidos a schwannoma, neurinoma, neurofibroma y tumor de las vainas nerviosas, por lo que el dato epidemiológico de la asociación o no de schwannoma intramedular y neurofibromatosis de uno y otro tipo no queda bien aclarado en la literatura, ni tampoco en este trabajo. En él se menciona la referencia bibliográfica de la descripción de 5 neurinomas intramedulares en pacientes afectos de neurofibromatosis tipo 1, algo prácticamente inverosímil, pero al acudir a la lectura de los artículos citados no resultan localizables las descripciones específicas de esos pacientes NF1, sino solamente referencias a su vez de otros autores que las han mencionado. Hechos como estos son los argumentos en los que se basó el comité de 
expertos sobre "neurinoma acústico", en $1993^{1}$, para recomendar que se utilice el término de schwannoma en vez de neurinoma, y de NF1 y NF2 en vez de neurofibromatosis o enfermedad de von Recklinghausen de forma genérica, recomendaciones aceptadas y seguidas en la nomenclatura actual por la OMS. En las revisiones histológicas de tumores raquídeos en bancos de tumores se ha comprobado que los neurofibromas se han observado exclusivamente en pacientes NF1 sin haber referencias intramedulares, y que los schwannomas raquídeos se han presentado de forma esporádica o en asociación con la NF2 ${ }^{3}$. Los pacientes con NF2 tienen, efectivamente, una alta tasa de tumores raquídeos, extramedulares e intramedulares. En estos últimos siempre ha destacado su carácter indolente, y en pocos casos se ha realizado extirpación quirúrgica y comprobación histológica, presumiendo que en su gran mayoría se trata de ependimomas de muy lenta evolución. Sin embargo, experiencias personales como la descrita en este artículo por los autores muestran cada vez más frecuentemente schwannomas intramedulares que, por su naturaleza benigna, tienen un comportamiento clínico muy poco agresivo y que, ante un planteamiento quirúrgico, son relativamente sencillos de extirpar si se conserva el plano de disección entre el tejido tumoral y el parénquima medular, no infiltrado.

Como corolario del artículo presentado, en mi opinión deberíamos añadir dos conclusiones: 1. Cuando se llega al diagnóstico de schwannoma tras la extirpación de un tumor intramedular, el paciente debería ser considerado "de riesgo" para la neurofibromatosis tipo 2 , y se le debería ofrecer un estudio de rastreo según protocolo ${ }^{2}$ para confirmar ó descartar la enfermedad, por su relevancia en el pronóstico y en la posible afectación de familiares. 2. Cuando se diagnostica un tumor intramedular en un paciente con neurofibromatosis tipo 2 conocida, se debe plantear el diagnóstico diferencial entre ependimoma y schwannoma, con matices distintos en el momento de un eventual tratamiento quirúrgico.

\section{Bibliografía}

1. Eldridge, R., Parry, D.: Vestibular schwannoma (acoustic neuroma) consensus development conference: summary. Neurosurgery 1992; 30: 962-964.

2. Evans, D.G.R., Baser, M.E., O'Reilly, B., et al.: Management of the patient and family with neurofibromatosis 2: a consensus conference statement. Br J Neurosurgery 2005; 19: $5-12$.

3. Halliday, A.L., Sobel, R.A., Martuza, R.L.: Benign spinal nerve sheath tumors: their occurrence sporadically and in neurofibromatosis types 1 and 2. J Neurosurg 1991; 74: 248-253.

J.M. de Campos Gutiérrez

Madrid 